\title{
Preconditioners based on windowed Fourier frames applied to elliptic partial differential equations
}

\author{
Samir K. Bhowmik • Christiaan C. Stolk
}

Received: 5 October 2010 / Accepted: 17 February 2011 / Published online: 3 March 2011

(C) The Author(s) 2011. This article is published with open access at Springerlink.com

\begin{abstract}
We investigate the application of windowed Fourier frames to the numerical solution of partial differential equations, focussing on elliptic equations. The action of a partial differential operator (PDO) on a windowed plane wave is close to a multiplication, where the multiplication factor is given by the symbol of the PDO evaluated at the wave number and central position of the windowed plane wave. This can be exploited in a preconditioning method for use in iterative inversion. For domains with periodic boundary conditions we find that the condition number with the preconditioning becomes bounded and the iteration converges well. For problems with a Dirichlet boundary condition, some large and small singular values remain. However the iterative inversion still appears to converge well.
\end{abstract}

Keywords Windowed Fourier frame $\cdot$ Symbol Elliptic PDE $\cdot$ Preconditioner

\section{Introduction}

Localization in the position-wave number space is an important concept in partial differential and other operator equations. The large class of pseudodifferential operators acts approximately local in the position-wave number space. Prominent examples

This research was funded by the Netherlands Organisation for Scientific Research through VIDI Grant 639.032 .509 .

S. K. Bhowmik ( $)$

Department of Mathematics, University of Dhaka, Dhaka 1000, Bangladesh

e-mail: bhowmiksk@gmail.com

C. C. Stolk

KdV Institute for Mathematics, University of Amsterdam, Amsterdam, The Netherlands e-mail: C.C.Stolk@uva.nl 
where this is put to use in numerics are wavelets and multigrid methods, methods that are closely related. Multigrid and wavelet approaches are very successful for elliptic problems, and have been applied also elsewhere. For wavelet concepts we refer to [7], the multigrid literature is very large, see e.g. the book [19].

Wavelets provide a decomposition in scale and position. However, they have little resolution in direction, i.e. the direction in the wave-number space. More recently, a frame of functions [14] called curvelets has been proposed as a tool for numerical analysis of PDEs [3]. Curvelets provide additional localization in direction. The localization in direction is better and better for the smaller scales by a so called parabolic scaling: A fourfold smaller scale leads to twice better resolution in direction and twice better resolution in position. A method of solving a pseudodifferential equation using curvelets, including a curvelet based approximation for the operator inverse suitable for use as a preconditioner was introducted in [12]. Approximations of pseudodifferential operators were discussed in [9].

In this paper we analyze windowed Fourier frames (WFFs), also referred to as Gabor frames. They provide a decomposition of the position-wave number space ("phase space") into rectangular blocks. Compared to curvelets it is a simpler decomposition, and easier to implement. Compared to wavelets it still offers better directional resolution, although at somewhat higher (log-linear) cost. WFFs are also simpler than curvelets in the sense that they are generated by translations and modulations of a single window function, while for curvelets there is no such set of transformations that exactly maps the one to the other.

Chan et al. [4] has considered elliptic problems and proposed circulant preconditioners to solve the resulting system of equations using iterative techniques, for example, the conjugate gradient method. They prove that such preconditioners could be chosen to reduce the condition number from $\mathcal{O}\left(n^{2}\right)$ to $\mathcal{O}(n)$, where $n$ grid points have been chosen to discretize the problem for a second order elliptic problem. Some popular preconditioning techniques to solve linear systems using iterative methods have been discussed in detail in [5], [7, Chap. 1], [11, Chap. 10], [15, Chap. 7] and references there in. These techniques includes use of positive definite matrices, incomplete $L U$ and cholesky factorizations, multilevel, multigrid, wavelet preconditioners and so on. It has been noticed that condition numbers are in control with most preconditioners and have slower growth compared with the unpreconditioned system.

We believe that, while multigrid and wavelets are very important as preconditioning methods, other possibilities should also be studied. The particular phase space localization of wavelets is well suited for elliptic equations, but not for other types of PDE, for example the Helmholtz equation. WFFs, curvelets or maybe other transforms are natural candidates to study in more general settings than the elliptic problem.

Our purpose is therefore to introduce a preconditioning method based on WFFs, and to establish that it performs well for certain discrete PDE's. We start with straightforward elliptic problems, and include an example where the different phase space localization properties provide an advantage for windowed Fourier preconditioning. For this study we consider a symmetric second order elliptic BVP with a finite and a periodic domains. We first discretize the PDE using a standard finite difference scheme. We use a preconditioner based on WFFs and the symbol of the operator 
while solving the discrete PDE using iterative linear solvers, to speed up the convergence.

The article is organized in the following way. We start by introducing the preconditioners in Sect. 2. We study boundedness and invertibility of a symmetrically preconditioned operator in Sect. 3. Some numerical test results are presented in Sect. 4. We finish this study in Sect. 5 with some concluding remarks.

\section{Preconditioners based on the symbol of the operator and a WFF}

In this section, we focus on introducing and defining the preconditioner based on the symbol of the partial differential operator and a WFF. As a model problem we consider the boundary value problem

$$
\mathcal{L} u=f \quad \text { in } \Omega
$$

with Dirichlet and periodic boundary conditions on $\partial \Omega$ where

$$
\mathcal{L} u=-\nabla \cdot(a(x) \nabla u)+b(x) u(x),
$$

for given real coefficients $a(x)$, and $b(x)$. Here we consider $\Omega \subset \mathbb{R}^{d}$, an open bounded domain, $f: \Omega \longrightarrow \mathbb{R}$ is a given function and $u: \Omega \cup \partial \Omega \longrightarrow \mathbb{R}$ is an unknown function. A detailed description of this type of problems can be found in $[2,10,16]$, and many references therein. We investigate both periodic and non-periodic boundary value problems.

We make the following assumptions on the coefficients, to ensure that $\mathcal{L}$ is boundedly invertible. We assume there is some constant $C$ such that $a(x) \geq C$. In the case of Dirichlet boundary conditions we consider $b \geq 0$, in the case of periodic boundary conditions we assume that $b \geq C_{0}>0$ for some constant $C_{0}$.

\section{Windowed Fourier frames}

We first give a short introduction of WFFs. Let $\mathbf{H}$ be a Hilbert space. A sequence $\left\{\psi_{n}\right\}_{n \in \Gamma}$ is a frame [14, Section 5.1.1, Definition 5.1] of $\mathbf{H}$ if there exist two constants $A>0, B>0$ such that for any $f \in \mathbf{H}$,

$$
A\|f\|^{2} \leq \sum_{n \in \Gamma}\left|\left\langle f, \psi_{n}\right\rangle\right|^{2} \leq B\|f\|^{2} .
$$

The index set $\Gamma$ might be finite or infinite and one can define a frame operator $F_{1}$ so that

$$
F_{1} f[n]=\left\langle f, \psi_{n}\right\rangle, \quad \text { for all } n \in \Gamma \text {. }
$$

If the condition (2) is satisfied then $F_{1}$ is called a frame operator. When $A=B$ the frame is said to be tight [14, page 155], [6]. 
Fig. 1 Example of a function from a windowed Fourier frame in one dimension with window function $w(x)=$ $\sin \left(\frac{\pi}{2} \frac{e^{-c / x}}{e^{-c / x}+e^{-c /(1-x)}}\right), c=1.5$

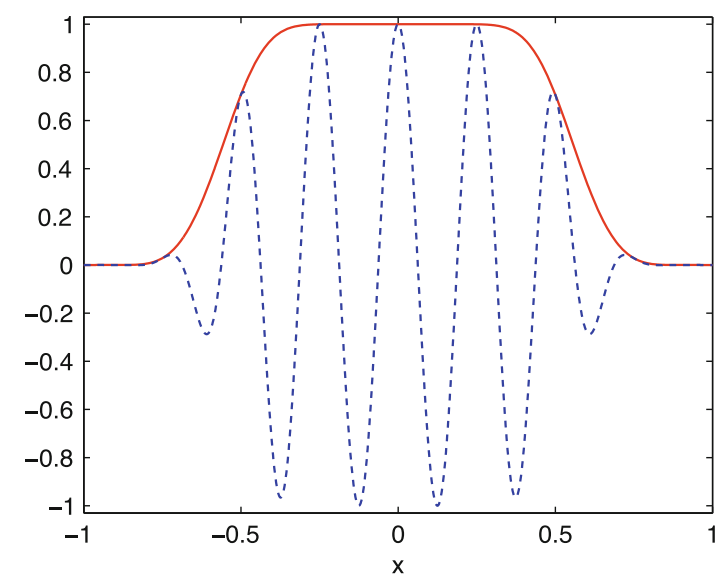

A window function is simply a function in $C_{0}^{\infty}\left(\mathbb{R}^{d}\right)$, i.e. it is smooth, and zero outside some chosen finite domain. Let us focus on $d=1$, and consider a real symmetric $(g(t)=g(-t)$, for all $t \in \mathbb{R})$, non-negative window function. We can translate $g$ by $v \in \mathbb{R}$ and modulate $g$ by frequency $\xi \in \mathbb{R}$ as $g_{v, \xi}(t)=e^{i \xi t} g(t-v)$, which are known as windowed Fourier atoms or Gabor atoms. Here $\left\|g_{v, \xi}(t)\right\|=1$ for any $v \in \mathbb{R}, \xi \in \mathbb{R}$ since $\|g\|=1$. If the functions $g_{v, \xi}(x)$ satisfy the frame condition (2), then they are called WFFs $\left[14\right.$, Section 5.4] and for any $f \in \mathbf{L}^{2}(\mathbb{R})$, the operator $F$ defined by

$$
F f(v, \xi)=\left\langle f, g_{v, \xi}\right\rangle=\int_{\mathbb{R}} f(t) g(t-v) e^{-i \xi t} d t
$$

is called the WFF operator. In Fig. 1 we present a sample WFF.

A version of the WFF transformation with discrete index set $\Gamma$, is obtained by restricting $(v, \xi)$ to a rectangular grid with interval size $v_{0}$ and $\xi_{0}$ in time and frequency respectively and define [14, page 182]

$$
g_{n, k}=g\left(t-n v_{0}\right) e^{i \xi_{k} t}
$$

where $\xi_{k}=k \xi_{0}$, which will be needed in Sect. 4 while implementing the preconditioners for elliptic PDE's. It is well understood $[8,14]$ that the windowed Fourier family $\left\{g_{n, k}\right\}_{(n, k) \in \mathbb{Z}^{2}}$ is a frame only if $\frac{2 \pi}{v_{0} \xi_{0}} \geq 1$ and the frame bounds $A$ and $B$ satisfies $A \leq \frac{2 \pi}{v_{0} \xi_{0}} \leq B$. Let $g$ be a window function with support $\left[-\frac{\pi}{\xi_{0}}, \frac{\pi}{\xi_{0}}\right]$. Then $\left\{g_{n, k}\right\}_{(n, k) \in \mathbb{Z}^{2}}$ is a tight frame [14] with a frame bound equal to $A$, if

$$
\frac{2 \pi}{\xi_{0}} \sum_{n=-\infty}^{\infty}\left|g\left(t-n v_{0}\right)\right|^{2}=A>0
$$

for all $t \in \mathbb{R}$. 
In the discrete setting, we replace the Fourier basis $\left\{e^{i k \xi_{0} t}\right\}_{k \in \mathbb{Z}}$ of $L^{2}\left[-\pi / \xi_{0}, \pi / \xi_{0}\right]$ by the discrete Fourier basis $\left\{e^{\frac{i 2 \pi k n}{N_{l}}}\right\}_{0 \leq k<N_{l}}$ of $\mathbb{C}^{N_{l}}$ to construct a discrete WFF. Let us consider $g[n]$ be a $N$ periodic discrete window function with a support and restricted to $[-N / 2, N / 2]$ that is included in $\left[-N_{l} / 2, N_{l} / 2-1\right]$. Then following [14] one can see that if $\bmod (N, M)=0$ and

$$
N_{l} \sum_{m=0}^{\frac{N}{M}-1}|g[n-m N]|^{2}=A>0 \quad \forall 0 \leq n<N
$$

then $\left\{g_{m, k}[n]=g[n-m M] e^{i 2 \pi k n / N_{l}}\right\}_{0 \leq k<N_{l}, 0 \leq m<K}$ is a tight frame in $\mathbb{C}^{N}$ with frame bound equal to $A$ where $K=\frac{N}{M}$. For a fixed window position indexed by $m$, the discrete WFF coefficients are

$$
F f[m, k]=\left\langle f, g_{m, k}\right\rangle=\sum_{n=0}^{N-1} f[n] g[n-m M] e^{-\frac{i 2 \pi k n}{N_{l}}},
$$

for all $0 \leq k<N_{l}$.

We set the length of the window equal to $2 v_{0}$, so that two successive windows have overlapping support and we choose tight WFFs. So we choose the parameters such that $\frac{2 \pi}{\xi_{0}}=2 v_{0}$, and $g$ has support in $\left[-v_{0}, v_{0}\right]$.

Several choices for the window function are discussed in Appendix. Our initial criterion for a good window function is the decay of its Fourier transform. We conclude that both the windows $h_{5}(x)$ with $c=1.5, d=0.9$ and the window formed by $h_{4}(x)$ are very well behaved. We experimented with both the windows to define the preconditioners and recorded the number of iterations needed for convergence while solving the precondition linear system. The numbers for these two choices of the window function are approximately equal, preconditioners formed by using the stretched window $h_{5}(x)$ with $c=1.5, d=0.9$ take a few iterations less than that of $h_{4}(x)$, but the effect is small. We decide to use the window $h_{5}(x)$ for this study since we designed it (and it performs a little better).

In order to apply WFFs to the BVP (1) we need to define and organise window functions for a multi-dimensional and bounded domain $\Omega \subset \mathbb{R}^{d}$, say $\bar{\Omega}=[a, b]^{d}$, where the domain can be both periodic and non-periodic. Multidimensional window functions are defined straightforwardly using a tensor product approach. For periodic domains we assume that the period is a discrete multiple of $v_{0}$, say $K v_{0}$. Then the set of coefficients becomes periodic with period $K$. In Sect. 3 we will discuss this further. For non-periodic problems, we define $K+1$ windows in each of the coordinate directions (instead of $K$ windows) with support $\frac{4 \pi}{K}$ (in each of the coordinate directions), considering an extended domain $\left[a-\frac{2 \pi}{K}, b+\frac{2 \pi}{K}\right]^{d} \supset \Omega$, by

$$
g_{j}(x)= \begin{cases}g\left(x-j v_{0}\right) & x \in \Omega, \\ 0 & x \notin \Omega,\end{cases}
$$


where $j \in\left\{1,2, \ldots,(K+1)^{d}\right\}$. Then we have $2^{d}(K+1),(d>1)$ windows that cross the boundary of $\Omega$.

\section{Preconditioners}

Here we return to our main discussion. First we give a short motivation why a PDO can be approximated by WFF's and the symbol of the PDO. For simplicity we start with BVP (1) in one dimension. Setting

$$
u=g_{j, k}(x)=e^{i \xi_{k} x} g\left(x-j v_{0}\right), \quad x \in \Omega \subset \mathbb{R},
$$

$j \in J$ (the set $J$ is defined in Sect. 3), $k \in \mathbb{Z}$, we get

$$
\begin{aligned}
a(x) \frac{\partial^{2} u}{\partial x^{2}}= & a(x) \frac{\partial}{\partial x}\left[i \xi_{k} e^{i \xi_{k} x} g\left(x-j v_{0}\right)+e^{i \xi_{k} x} \frac{d}{d x} g\left(x-j v_{0}\right)\right] \\
= & a(x)\left(-\xi_{k}^{2} e^{i \xi_{k} x} g\left(x-j v_{0}\right)+\left(1+i \xi_{k}\right) e^{i \xi_{k} x} \frac{d}{d x} g\left(x-j v_{0}\right)\right. \\
& \left.+e^{i \xi_{k} x} \frac{d^{2}}{d x^{2}} g\left(x-j v_{0}\right)\right) \\
= & A_{1}+B+C,
\end{aligned}
$$

where $A_{1}$ is the leading part (when $\xi_{k}$ is very large) of the elliptic operator acting on (1). When $\xi_{k}$ is very large, $\xi_{k}^{2}$ is the dominating term in $a(x) \frac{d^{2}}{d x^{2}}$ and so the differential operator $a(x) \frac{d^{2}}{d x^{2}}$ can be approximated by a multiplication operator $a(x) \xi_{k}^{2}$. Now let us represent a function $u(x) \in \mathbf{H}$ using WFFs

$$
u(x)=\sum_{j, k} C_{j, k} g_{j, k}(x), \quad x \in \Omega
$$

where

$$
C_{j, k}=\left\langle u(x), g_{j, k}(x)\right\rangle \text { and } g_{j, k}(x)=e^{i \xi_{k} x} g\left(x-j v_{0}\right) .
$$

Then we can approximate

$$
a(x) \frac{\partial^{2} u}{\partial x^{2}} \approx \sum a(x) \xi_{k}^{2} C_{j, k} g_{j, k}(x)=\sum a(x) \xi_{k}^{2}\left\langle u, g_{j, k}\right\rangle g_{j, k}
$$

Here we observe that the PDO $\left(a(x) \frac{\partial^{2} u}{\partial x^{2}}\right)$ can be approximately reconstructed by the tight WFFs and its duals multiplied by the symbol of the PDO if $a$ varies little over the support of $g_{j, k}$. This result motivates us to define preconditioners based on symbols of the PDO's and WFF's.

It is to note that we consider $\bar{x}_{j}, j \in J$, as midpoints of the subdomains of $\Omega$. To bound the condition number and to speed up the convergence of iterative solvers 
we are interested in defining preconditioners for a discrete equivalent of (1) based on the symbol and WFFs. To this end, we define an invertible matrix $M$

$$
M_{\tilde{j}, \tilde{k} ; j, k}=\delta_{\tilde{j}, j} \delta_{\tilde{k}, k}\left(1+a\left(\bar{x}_{j}\right) \xi_{k}^{2}\right)
$$

We solve an equivalent system (of $\mathcal{A} u=f$, in which $\mathcal{A}$ is a symmetric discrete equivalent of the operator acting on (1) using a finite difference scheme). Several forms of preconditioning can be distinguished:

Symmetric preconditioning $P \mathcal{A} P \tilde{u}=P f$, coupled with $P \tilde{u}=u$, where $P=$ $F^{*} M^{-\frac{1}{2}} F$.

Left preconditioning $P \mathcal{A} u=P f$, where $P=F^{*} M^{-1} F$.

Right preconditioning $\mathcal{A} P \tilde{u}=f$, coupled with $u=P \tilde{u}$ where $P=F^{*} M^{-1} F$.

In each case $F^{*}$ is the conjugate transpose of the frame operator $F$. The relation between the left and right preconditioners is established in the following result.

Theorem 2.1 The singular values of the left preconditioned matrix $P \mathcal{A}$ and the right preconditioned matrix $\mathcal{A} P$ are equal where $P=F^{*} M^{-1} F$.

Proof We have

$$
(P \mathcal{A})^{*}=\mathcal{A}^{*} P^{*}=\mathcal{A} P,
$$

since $\mathcal{A}$ is symmetric and

$$
P^{*}=\left(F^{*} M^{-1} F\right)^{*}=\left(M^{-1} F\right)^{*} F=F^{*} M^{-1} F,
$$

proves the claim since singular values of $P \mathcal{A}$ and $(P \mathcal{A})^{*}$ are the same.

\section{Boundedness and invertibility of the symmetrically preconditioned operator $\boldsymbol{P} \mathcal{L} P$}

A key property of a preconditioner, is that the condition number of the preconditioned operator is bounded independent of the discretisation. We will show that the symmetrically preconditioned operator $P \mathcal{L} P$, where $P=F^{*} M^{-1 / 2} F$ and $F$ stands for tight WFF operator, considered in the continuous case, defines a continuous map, with continuous inverse on $L^{2}(\Omega)$. Under a suitable discretisation this can be used to derive the first mentioned property, although we will not do so (instead we study some discrete systems numerically in the next section). We prove this in the case of the domain

$$
\Omega=[0,2 \pi)^{n}=\mathbb{T}^{n}
$$

i.e. $[0,2 \pi]^{n}$ with the periodic boundary conditions. In the case of a domain with a boundary we will see in the section on numerics that there are a few singular values 
that grow if the discretisation parameter becomes small, so that the result appears to be false.

Theorem 3.1 The self adjoint operator $P \mathcal{L} P: L_{2}(\Omega) \rightarrow L_{2}(\Omega)$ is boundedly invertible, that is, there exists $c_{2} \geq c_{1}>0$ such that

$$
c_{1}\|u\|^{2} \leq\langle P \mathcal{L} P u, u\rangle \leq c_{2}\|u\|^{2}, \text { for all } u \in H .
$$

By assumption $\mathcal{L}: H^{1} \rightarrow H^{-1}$ is boundedly invertible. Therefore, to establish Theorem 3.1, it is sufficient to show that $P$ is boundedly invertible from $L_{2}(\Omega)$ to $H^{1}(\Omega)$, and that $P$ is boundedly invertible from $H^{-1}(\Omega)$ to $L_{2}(\Omega)$. In fact it sufficient to show that $P$ is boundedly invertible from $L_{2}(\Omega)$ to $H^{1}(\Omega)$, as this implies that $P^{*}$ is boundedly invertible from $H^{-1}(\Omega)$ to $L_{2}(\Omega)$ and $P=P^{*}$.

One may think of $\mathbb{T}^{n}$ as the hypercube $\left[0,2 \pi\left[{ }^{n} \subset \mathbb{R}^{n}\right.\right.$ or $\left[-\pi, \pi\left[{ }^{n}\right.\right.$. Functions on $\mathbb{T}^{n}$ may be thought as those functions on $\mathbb{R}^{n}$ that are $2 \pi$ periodic in each of the coordinate directions.Let $p \in \mathbb{R}$. The sobolev space $H^{p}(\Omega)$ is the space of all functions $\psi \in L^{2}(\Omega)$ that satisfy

$$
\|\psi\|_{p}^{2}=\sum_{m \in \mathbb{Z}^{n}}\left(1+|m|^{2}\right)^{p}\left|a_{m}\right|^{2}<\infty
$$

for the Fourier coefficients $a_{m}$ of $\psi$. The space $H^{p}(\Omega)$ is a Hilbert space with the scalar product defined by

$$
\langle\phi, \psi\rangle_{p}:=\sum_{m \in \mathbb{Z}^{n}}\left(1+|m|^{2}\right)^{p} a_{m} \bar{b}_{m}
$$

for $\phi, \psi \in H^{p}(\Omega)$ with Fourier coefficients $a_{m}$ and $b_{m}$, respectively.

When we consider one window only, the WFF operator $F$ becomes the Fourier transform operator. So we first prove the invertibility of the operator $P \mathcal{L} P$ considering $F$ as the Fourier transform operator. From here we denote $M_{\frac{1}{2}}=M^{-\frac{1}{2}}$. We first show that $P u \in H^{1}(\Omega)$ for $u \in L_{2}(\Omega)$. Here

$$
\langle P u, P u\rangle=\left\langle F^{-1} M_{\frac{1}{2}} F u, F^{-1} M_{\frac{1}{2}} F u\right\rangle=\left\langle M_{\frac{1}{2}} F u, M_{\frac{1}{2}} F u\right\rangle
$$

where $F u \in \ell_{2}(\mathbb{Z})$ is the sequence of Fourier coefficients of $u \in L_{2}(\Omega)$. Now using (6) we have

$$
\langle P u, P u\rangle_{1}=\sum_{m \in \mathbb{Z}^{n}}\left(1+|m|^{2}\right)\left(M_{\frac{1}{2}}\right)^{2}\left|(F u)_{m}\right|^{2}
$$

where $(F u)_{m} \in \ell_{2}(\mathbb{Z})$ are the Fourier coefficients of $u \in L_{2}(\Omega)$. Now for any $m \in \mathbb{Z}^{n}$ there exists $0<\tilde{A}<\tilde{B}$ such that $\tilde{A} \leq\left(1+|m|^{2}\right)\left(M_{\frac{1}{2}}\right)^{2} \leq \tilde{B}$. So one gets

$$
\tilde{A}\|u\|^{2} \leq\|P u\|_{1} \leq \tilde{B}\|u\|^{2},
$$


and

$$
P u=F^{-1} M_{\frac{1}{2}} F u \in H^{1}(\Omega), \quad \text { if } u \in L_{2}(\Omega) .
$$

Thus there exists $0<c_{1} \leq c_{2}$ such that

$$
c_{1}\|u\|^{2} \leq\langle P \mathcal{L} P u, u\rangle \leq c_{2}\|u\|^{2},
$$

and that confirms the boundedly invertibility of $P \mathcal{L} P: L_{2}(\Omega) \rightarrow L_{2}(\Omega)$, since $\mathcal{L}$ is boundedly invertible and $P^{*}=P$.

Next we investigate the boundedly invertibility of $P \mathcal{L} P: L_{2}(\Omega) \rightarrow L_{2}(\Omega)$ considering $F$ as a WFF operator. To define window functions we consider $K$ uniform subintervals of size $\frac{2 \pi}{K}$ in each of the coordinate directions so that we can divide $\Omega\left(=\mathbb{T}^{n}\right)$ into $K^{n}$ subdomains. Then we define $K^{n}$ overlapping subdomains with length $\frac{4 \pi}{K}$ on each of the coordinate directions and denote them by $D_{j} \subset \Omega$ for all $j \in J$ where $J=\{1,2, \ldots, \tilde{K}\}$ with $\tilde{K}=K^{n}$. We consider $K$ windows on each of the coordinate directions. We define window functions $g_{j}(x)$ on $\Omega$ for all $j \in J$ satisfying $\sum_{j \in J} g_{j}^{2}(x)=1$; where $x \in \Omega$. The support of each $g_{j}(x)$ is contained in $D_{j}$. By $x_{j}$ we denote the midpoint of the block $D_{j}$. Of course for the periodic setting one needs to consider appropriate boundary domains, in one dimension for example, $D_{1}=2 \pi\left[0, \frac{1}{K}\right] \cup 2 \pi\left[\frac{K-1}{K}, 1\right)$. We will also assume that the support of $g_{j}$ stays away some small distance from the boundaries. The windowing operator is denoted by $W$, defined by

$$
\begin{gathered}
W: L^{2}(\Omega) \longrightarrow L^{2}\left(D_{1}\right) \times L^{2}\left(D_{2}\right) \times \cdots \times L^{2}\left(D_{\tilde{K}}\right) \\
W u=\left(g_{1} u, g_{2} u, \ldots, g_{\tilde{K}} u\right) .
\end{gathered}
$$

( $W u$ can also be viewed as an element of the larger space $L^{2}\left(\Omega, C^{\tilde{K}}\right)$.) The adjoint windowing operator $W^{*}$ then equals

$$
W^{*}\left(u_{1}, u_{2}, u_{3}, \ldots, u_{\tilde{K}}\right)=\sum_{j \in J} g_{j} u_{j} .
$$

The Fourier transform of the windowed Fourier transform is done on each domain $D_{j}$. We will write $\mathcal{F}_{D_{j}}$ for this Fourier transform on functions restricted to $D_{j}$. We redefine the operator $P$ as

$$
P=\sum_{j \in J} g_{j}\left(\mathcal{F}_{D_{j}}^{-1} M_{\frac{1}{2}} \mathcal{F}_{D_{j}}\right) g_{j}
$$

We first study $P$ as an operator $H^{1 / 2} \rightarrow H^{-1 / 2}$, starting with a result about $W$. 
Lemma 3.2 Let $W$ be defined by (8). Then there exists $0<\alpha<\beta$ such that

$$
\alpha\|W u\|_{H^{1 / 2}\left(\Omega, C^{\tilde{K}}\right)} \leq\|u\|_{H^{1 / 2}(\Omega, C)} \leq \beta\|W u\|_{H^{1 / 2}\left(\Omega, C^{\tilde{K}}\right)} .
$$

Proof We consider separately the two inequalities

$$
\alpha\|W u\|_{H^{1 / 2}\left(\Omega, C^{\tilde{K}}\right)} \leq\|u\|_{H^{1 / 2}(\Omega, C)}
$$

and

$$
\|u\|_{H^{1 / 2}(\Omega, C)} \leq \beta\|W u\|_{H^{1 / 2}\left(\Omega, C^{\tilde{K}}\right)} .
$$

Equation (10) is easy, it follows directly from the continuity of the multiplication by $g_{j}$ on $H^{1 / 2}$. Equation (11) is more difficult. We solve it as follows. Define $E_{(s)}$ to be the pseudodifferential on $\Omega\left(=\mathbb{T}^{n}\right.$, the torus) with symbol $\left(1+\|\xi\|^{2}\right)^{s / 2}$. This defines a self adjoint operator. The $H^{s}$ norm of a function $u$ is equivalent to $\left\|E_{(s)} u\right\|_{L_{2}}$. In this proof we in fact use this formula for the norm.

We start with the basic estimate

$$
\|W u\|_{H^{1 / 2}}^{2} \geq \frac{1}{2}\|W u\|_{L_{2}}^{2}+\frac{1}{C}\left\|E_{(1 / 2)} W u\right\|^{2}
$$

where $C \geq 2$ is a constant to be chosen later. For $\|W u\|_{L_{2}}^{2}$ we find the following

$$
\|u\|_{L^{2}}^{2}=\left\langle u, \sum_{j} g_{j}^{2} u\right\rangle=\sum_{j}\left\langle g_{j} u, g_{j} u\right\rangle=\|W u\|_{L_{2}}^{2} .
$$

For $\left\|E_{(1 / 2)} W u\right\|^{2}$ we use the following

$$
\begin{aligned}
\left\|E_{(1 / 2)} W u\right\|_{L_{2}}^{2} & =\sum_{j}\left\langle E_{(1 / 2)} g_{j} u, E_{(1 / 2)} g_{j} u\right\rangle \\
& =\left\langle E_{(1 / 2)} u, E_{(1 / 2)} \sum_{j} g_{j}^{2} u\right\rangle+\sum_{j}\left\langle u,\left[E_{(1)}, g_{j}\right] g_{j} u\right\rangle, \\
& =\|u\|_{H^{1 / 2}}^{2}+\sum_{j}\left\langle u,\left[E_{(1)}, g_{j}\right] g_{j} u\right\rangle,
\end{aligned}
$$

where we used that $E_{(1 / 2)} E_{(1 / 2)}=E_{(1)}$ and $\left[E_{(1)}, g_{j}\right]=E_{(1)} g_{j}-g_{j} E_{(1)}$. The operator $\left[E_{(1)}, g_{j}\right]$ is a pseudodifferential operator of order 0 (follows from [1]). Therefore we find that there is a constant $D$ such that

$$
\left|\sum_{j}\left\langle u,\left[E_{(1)}, g_{j}\right] g_{j} u\right\rangle\right| \leq D\|u\|_{L_{2}}^{2} .
$$


We now use what we just described in (12) and we choose $C=\min (2 D, 2)$. This yields

$$
\begin{aligned}
\|W u\|_{H^{1 / 2}}^{2} & \geq \frac{1}{2}\|u\|_{L_{2}}^{2}+\frac{1}{C}\left(\left\|E_{(1 / 2)} u\right\|_{L_{2}}^{2}-D\|u\|_{L_{2}}^{2}\right) \\
& \geq \frac{1}{C}\left\|E_{(1 / 2)} u\right\|_{L_{2}}^{2}=\frac{1}{C}\|u\|_{H^{1 / 2}}^{2} .
\end{aligned}
$$

We therefore have proved (11).

Using the lemma we can prove the following

Proposition 3.3 $P$ is boundedly invertible $H^{-1 / 2}(\Omega) \rightarrow H^{1 / 2}(\Omega)$.

Proof The operator $W$ maps from $H^{1 / 2}(\Omega)$ to the Sobolev space $H^{1 / 2}\left(D_{1}\right) \times$ $H^{1 / 2}\left(D_{2}\right) \times \cdots \times H^{1 / 2}\left(D_{\tilde{K}}\right)$. The proposition follows from the lemma we just proved and the Lax-Milgram theorem, using the Fourier coefficient description of this Sobolev spaces.

Next we study $P$ as an operator $L^{2}(\Omega) \rightarrow H^{-1}(\Omega)$, by first proving that $P$ is a pseudodifferential operator.

Proposition 3.4 If the window functions $g_{j}(x) \in C_{0}^{\infty}\left(D_{j}\right)$, then the operator $P$ defined by

$$
P=\sum_{j \in J} P_{j}=\sum_{j \in J} g_{j} S_{j} g_{j}
$$

is a periodic elliptic $P$ sDO of order -1 where $S_{j}=\mathcal{F}_{D_{j}}^{-1} M_{1 / 2} \mathcal{F}_{D_{j}}$ is a periodic elliptic PsDO of order -1 .

Before we go to prove the proposition, we will need some definitions and results about periodic pseudodifferential operators $[18,20]$. Let $\mathcal{D}\left(\mathbb{T}^{n}\right)$ be the vector space $C^{\infty}\left(\mathbb{T}^{n}\right)$ endowed with the usual test function topology. Then any continuous linear operator $A: \mathcal{D}\left(\mathbb{T}^{n}\right) \rightarrow \mathcal{D}\left(\mathbb{T}^{n}\right)$ can be represented as

$$
(A u)(x)=\sum_{\xi \in \mathbb{Z}^{n}} \sigma_{A}(x, \xi) \hat{u}(\xi) e^{i x \cdot \xi},
$$

where

$$
\sigma_{A}(x, \xi)=e^{-i x \cdot \xi} A e^{i x \cdot \xi}
$$

is called the symbol of the operator $A$. Let $m \in \mathbb{R}$ and $0 \leq \delta<\rho \leq 1$. In our case $\delta=0$ and $\rho=1$. An operator defined by (13) is called a periodic pseudodifferential operator (PsDO) of order $\alpha$ if the unique function $\sigma_{A} \in C^{\infty}\left(\mathbb{T}^{n} \times \mathbb{Z}^{n}\right)$ defined by (14) satisfies

$$
\left|\Delta_{\xi}^{\alpha} \partial_{x}^{\beta} \sigma_{A}(x, \xi)\right| \leq C_{\sigma \alpha \beta m}\langle\xi\rangle^{m-\rho|\alpha|+\delta|\beta|}
$$


for every $x \in \mathbb{T}^{n}$, for every $\alpha, \beta \in \mathbb{N}^{n}$ and $\langle\xi\rangle:=\left(1+|\xi|^{2}\right)^{1 / 2}$. Here by $S_{\rho, \delta}^{m}\left(\mathbb{T}^{n} \times\right.$ $\left.\mathbb{Z}^{n}\right)$ we denote the space of functions $\sigma_{A} \in C^{\infty}\left(\mathbb{T}^{n} \times \mathbb{Z}^{n}\right)$ that satisfies (15). If $\sigma_{A} \in S_{\rho, \delta}^{m}\left(\mathbb{T}^{n} \times \mathbb{Z}^{n}\right)$, one may denote $A \in \mathrm{Op} S_{\rho, \delta}^{m}\left(\mathbb{T}^{n} \times \mathbb{Z}^{n}\right)$. An operator $A \in$ Op $S_{\rho, \delta}^{m}\left(\mathbb{T}^{n} \times \mathbb{Z}^{n}\right)$ is called elliptic if, in addition, the symbol $\sigma_{A} \in S_{\rho, \delta}^{m}\left(\mathbb{T}^{n} \times \mathbb{Z}^{n}\right)$ of $A$ satisfies $\left|\sigma_{A}(x, \xi)\right| \geq C|\xi|^{m}$ where $x \in \mathbb{R}^{n}, C>0$ and $|\xi| \geq \xi_{0} \geq 0$.

Proof of proposition 3.4 First we show that the operator $S_{j}=\mathcal{F}_{D_{j}}^{-1} M_{1 / 2} \mathcal{F}_{D_{j}}$ is a periodic elliptic PsDO with period $\frac{4 \pi}{K}$ on each of the coordinate directions. Now for any $u \in \mathcal{D}\left(\mathbb{T}^{n}\right)$ we have $S_{j} u=\mathcal{F}_{D_{j}}^{-1}\left(M_{1 / 2}\left(\mathcal{F}_{D_{j}} u(\xi)\right)\right)$, with symbol $a_{j}(\xi)=M_{\frac{1}{2}}(\xi)=$ $\frac{1}{\sqrt{1+\xi^{2}}}$. Consider $v_{k} \in \mathbb{Z}^{n}$ with $\left(v_{k}\right)_{k}=1$ and $\left(v_{k}\right)_{i}=0$ if $i \neq k$. Now using difference calculus

$$
\begin{aligned}
\Delta_{\xi_{k}}^{1} M_{\frac{1}{2}}(\xi) & =M_{\frac{1}{2}}\left(\xi+v_{k}\right)-M_{\frac{1}{2}}(\xi) \\
& =\left(1+a\left(x_{j}\right)\left(\xi+v_{k}\right)^{2}\right)^{-1 / 2}-\left(1+a\left(x_{j}\right) \xi^{2}\right)^{-1 / 2}
\end{aligned}
$$

gives

$$
\left|\Delta_{\xi_{k}}^{1} M_{\frac{1}{2}}(\xi)\right| \leq C(1+|\xi|)^{-2},
$$

and similarly for higher order differences, and thus $M_{\frac{1}{2}}(\xi) \in S_{\rho, \delta}^{-1}\left(\mathbb{T}^{n} \times \mathbb{Z}^{n}\right)[18,20]$. That is to say that $S_{j}$ is a periodic elliptic PsDO of order $m=-1$ with period $\frac{4 \pi}{K}$ on each of the coordinate directions (using the binomial theorem and the Leibnitz formula for differences [18] one can deduce the similar relation for any $\alpha$ ). The previous statement implies that the distribution kernel of $S_{j}$, let us denote it here by $K(x, y)$, has singularities at $x=y+\frac{2 \pi}{K} k$, where $k \in \mathbb{Z}^{n}$. Therefore, when it is restricted to $x \in \operatorname{supp}\left(g_{j}\right), y \in \operatorname{supp}\left(g_{j}\right)$, then the singularities are contained in the set $x=y$.

Then we show that $P_{j}=g_{j}(x) S_{j}(x, \xi) g_{j}(x)$ is a PsDO for all $j \in J$. Here we consider $g_{j}(x) \in C_{0}^{\infty}\left(D_{j}\right), j \in J$, and thus $g_{j}$ is a PsDO of order 0 . Then it follows from $[13,21]$ that the composition $S_{j} g_{j}$ is a periodic elliptic PsDO of order -1 , and so is the operator (since $S_{j}$ is a PsDO of order -1) $P_{j}$ with principal symbol $\sigma_{j}(x, \xi)=$ $g_{j}(x) a_{j}(\xi) g_{j}(x)$. Since $g_{j}(x) \in C_{0}^{\infty}\left(D_{j}\right)$, it can be viewed as $g_{j}(x) \in C_{0}^{\infty}(\Omega)$. Thus for all $j \in J$ the symbols $\sigma_{j}(x, \xi)$ can be extended to $\sigma_{j} \in S_{\rho, \delta}^{-1}\left(\Omega \times \mathbb{Z}^{n}\right)$.

We have $P=\sum_{j \in J} g_{j} S_{j} g_{j}$. Here $g_{j} S_{j} g_{j}, j \in J$ are periodic elliptic PsDOs of order -1 . Let us denote the principal symbol of $S_{j}$ by $a_{j}$. So it follows that $P$ is a periodic elliptic PsDO of order -1 since $P$ is a sum of PsDOs of order -1 .

Since $P$ is an elliptic PsDO, it follows from $[13,21]$ and references there in that there is a parametrix $Q$ such that $Q P=I+R$ with $R$ a smoothing operator. For our purposes it is sufficient that $R$ is a PsDO of order -1 (see PsDO literature, parametrix, for example [1, page 18]). Let us now discuss the kernel of the operator $P$. The kernel of $P$ is contained in the kernel of $Q P$. The operator $R$ is compact. We work with operators on $L_{2}$, so it is an operator from $L_{2}$ to $H^{1}$, and therefore compact as an operator on $L_{2}$. But since this is PsDO theory, we can also work on operators on $H^{s}$, 
and then $R$ is continuous $H^{s} \rightarrow H^{s+1}$ and compact as an operator on $H^{s}$. From the theory of compact operators, it follows that, for any $C>0$, there is at most a finite dimensional set of vectors in $L_{2}$, such that $\|R u\|_{L_{2}} \geq C\|u\|$ (e.g. set $C=1 / 2$.) Then it shows also that the kernel of $P Q$ is finite dimensional and hence that the kernel of $P$ is finite dimensional. Similarly the cokernel of $P$, which is defined as the kernel of $P$ is finite dimensional. We claim that the elements of $\operatorname{ker}(Q P)$ are in $C^{\infty}$. The elements of $\operatorname{ker}(Q P)$ satisfy $u=-R u$. Let us say $u \in L_{2}$. Then $R u \in H^{1}$ because $R$ is a PsDO of order -1 . But $u=-R u$, so in fact $u \in H^{1}$. But then $R u \in H^{2}$, because $R$ is continuous $H^{1} \rightarrow H^{2}$. And so forth. It follows that $u \in C^{\infty}$. Since we already proved in Proposition 3.3 that $P$ is invertible $H^{-1 / 2} \rightarrow H^{1 / 2}$, it follows that the kernel and cokernel of $P$ are the zero sets and that $P$ is invertible $H^{s} \rightarrow H^{s+1}, s \in \mathbb{R}$.

Next we study the inverse $P^{-1}$ and show that it is a PsDO. As $P$ is an elliptic PsDO, there exists $Q \in \mathrm{Op}\left(S^{1}\right)[13,21]$ such that $Q P=I+R$ and $P Q=I+\tilde{R}$, where $R \in \mathrm{Op}\left(S^{-\infty}\right)$ and $\tilde{R} \in \mathrm{Op}\left(S^{-\infty}\right)$. Let us consider $V: H^{1 / 2} \rightarrow H^{-1 / 2}$ be the inverse of $P: H^{-1 / 2} \rightarrow H^{1 / 2}$. Then one gets

$$
Q P V=(I+R) V
$$

and

$$
Q P V=Q
$$

Combining (16) and (17) we have $(I+R) V=Q$ and so

$$
Q-V=R V
$$

Also one can write

$$
V P Q=Q
$$

and

$$
V P Q=V(I+\tilde{R})
$$

Combining (19), (20) and (18) one gets

$$
Q-V=V \tilde{R}=(Q+(V-Q)) \tilde{R}=Q \tilde{R}-R V \tilde{R} \in O P\left(S^{-\infty}\right),
$$

and so $V \in O P\left(S^{1}\right)$.

From here we may conclude that Theorem 3.1 is proved. However, there is no estimate of $c_{1}$, so we add a slightly more direct estimate for the constant $c_{1}$.

Proposition 3.5 There are constants $0<A \leq B$ such that

$$
A\|P u\|_{H^{1}} \leq\|u\|_{L_{2}} \leq B\|P u\|_{H^{1}}, \quad \text { for any } u \in L_{2}(\Omega) \text {. }
$$


Proof The second inequality follows from the fact that $P$ is a pseudodifferential operator of order 1 . For the first inequality consider the family of pseudodifferential operators $E_{\lambda, s}$, with symbol $E_{\lambda, s}(x, \xi)=\left(1+\frac{\xi^{2}}{\lambda^{2}}\right)^{s / 2}$. Clearly $P u$ satisfies

$$
\begin{aligned}
\|P u\|_{H^{1}} & \geq\left\|E_{\lambda, 1 / 2} P u\right\|_{H^{1 / 2}}=\left\|P E_{\lambda, 1 / 2} u+\left[E_{\lambda, 1 / 2}, P\right] u\right\|_{H^{1 / 2}} \\
& \geq\left\|P E_{\lambda, 1 / 2} u\right\|_{H^{1 / 2}}-\left\|\left[E_{\lambda, 1 / 2}, P\right] u\right\|_{H^{1 / 2}} .
\end{aligned}
$$

The first term can be estimated by

$$
\left\|P E_{\lambda, 1 / 2} u\right\|_{H^{1 / 2}} \geq \sqrt{\lambda}\|P\|_{H^{-1 / 2} \rightarrow H^{1 / 2}}\|u\|_{L^{2}} .
$$

The second term can be written as

$$
\sum_{j}\left[E_{\lambda, 1 / 2}, g_{j} S_{j} g_{j}\right]=\sum_{j}\left[E_{\lambda, 1 / 2}, g_{j}\right] S_{j} g_{j}+g_{j} S_{j}\left[E_{\lambda, 1 / 2}, g_{j}\right]
$$

The commutator $\left[E_{\lambda, 1 / 2}, g_{j}\right]$ is a pseudodifferential operator whose symbol can estimated in terms of $\lambda$, it is given by an asymptotic sum of the form

$$
\sum_{j=0}^{\infty} \frac{1}{\lambda^{j+1}} B_{j}\left(x, \frac{\xi}{\lambda}\right)
$$

where the $B_{j}$ are symbols of order $-1 / 2-j$. It follows that we have the estimate

$$
\left\|\left[E_{\lambda, 1 / 2}, g_{j}\right]\right\|_{H^{s} \rightarrow H^{s}} \leq C \lambda^{-1}
$$

Equations (23) and (24) show that the second term on the r.h.s. of (22) can be estimated by $C \lambda^{-1}\|u\|_{L^{2}}$. By choosing $\lambda$ large enough, it follows that the first inequality of (21) holds.

\section{Numerical experiments}

Now the question arises how efficient it is to solve constant and variable coefficient PDEs using the proposed preconditioner? Does the preconditioned system converge faster than the unpreconditioned system while using some iterative solvers for linear system of equations, for example, the conjugate gradient method? To investigate it, we experiment our technique focusing elliptic BVPs and compare it with the conjugate gradient method within the framework of MATLAB. 
4.1 One dimensional examples

Let us start with a one dimensional case $(d=1)$. Then the BVP $(1)$ takes the form

$$
-\frac{d}{d x}\left(a(x) \frac{d u(x)}{d x}\right)+b(x) u(x)=f(x), \quad \forall x \in \Omega \subset \mathbb{R}
$$

with

$$
u=0 \quad \text { on } \partial \Omega
$$

where $a(x)$, and $b(x)$ are known functions of $x$ with $a(x) \neq 0$. We approximate the problem $\mathcal{L} u=f$ using a finite difference scheme. Let us define the grids as $\hat{x}_{j}=j h, j=0,1,2, \ldots, N$ with grid spacing $h$, and $N \geq 2$. We define the approximations to the solution $u$ by $u_{j}$ defined on the grid points $\hat{x}_{j}$ for all $j=0,1,2, \ldots, N$. The BVP (25) can be approximated by

$$
-\frac{1}{h^{2}}\left(a_{i+\frac{1}{2}} u_{i+1}-u_{i}\left(a_{i+\frac{1}{2}}+a_{i-\frac{1}{2}}\right)+a_{i-\frac{1}{2}} u_{i-1}\right)+b_{i} u_{i}=f_{i}
$$

for all $i=1,2, \ldots, N$ where $a_{i}=a\left(\hat{x}_{i}\right), u_{i}=u\left(\hat{x}_{i}\right), b_{i}=b\left(\hat{x}_{i}\right), f_{i}=f\left(\hat{x}_{i}\right)$, $a_{i \pm \frac{1}{2}}=\frac{a_{i}+a_{i \pm 1}}{2}$ and using boundary condition $u_{0}=u_{N}=0$. We write (26) in the matrix form as $\mathcal{A} u=f$. In Fig. 2, we plot spectral radius and condition numbers of $\mathcal{A}$ for several choices of system size to demonstrate the polynomial growth of condition numbers. It is noticed from Fig. 2 (also several articles and books on finite difference schemes have a clear discussion about the eigenvalues of $\mathcal{A}$, e.g., $[2,15,17]$ and references therein) that the condition number of a matrix $\mathcal{A}$ grows like $\mathcal{O}\left(1 / h^{2}\right)$ (when $a(x)=1$, and as a result the convergence of any iterative methods become slower.

We consider three examples to address the questions arise in our previous discussion. In the first example, we consider $a(x)=1, b(x)=0$ and observe singular values and condition numbers of the symmetric and left preconditioned matrices. We also compare the number of iterations taken by the preconditioned solvers to converge. Then we repeat our experiments when $a(x)$ varies with $x$ in the second example. Here we compare condition numbers by varying number of windows (to cover the domain) to show the efficiency of windowing. We also show the advantage of using the preconditioned CG solver by comparing the number of iterations with the CG solver. In the third example the coefficient varies discontinuously.

Example 4.1 Consider the BVP

$$
-\frac{d^{2}}{d x^{2}} u(x)=f(x), \quad \forall 0<x<1,
$$

with boundary conditions

1. $u(0)=0$ and $u(1)=0$.

2. periodic boundary condition $u(0)=u(1)$. 


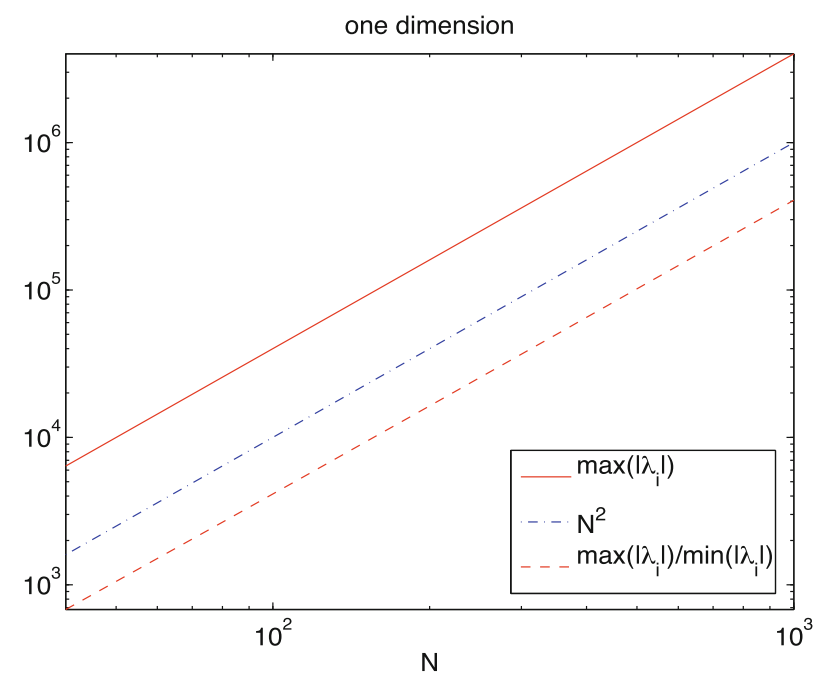

Fig. 2 We show spectral radius, and condition number of the matrix operator acting on (26) for various choices of system sizes with $a=1$ and $b=0$

The discrete operator $\mathcal{A}$ can be found in (26), to enforce the periodicity, when needed, we define $\mathcal{A}(N, 1)=\mathcal{A}(1, N)=-\frac{1}{h^{2}}$. In Fig. 3 we compare singular values of $P \mathcal{A} P$ and $P \mathcal{A}$ with the singular values of $\mathcal{A}$ (with periodic and non-periodic settings). From these computations, we notice that most of the singular values are close to one, whereas the singular values/eigenvalues of $\mathcal{A}$ are [15, page 561]

$$
\lambda_{j}=\frac{4}{h^{2}} \sin ^{2}\left(\frac{j \pi}{2(N+1)}\right), \quad \forall j=1,2, \ldots, N
$$

In the periodic case there is a singular value zero due to the constant solutions.

In Figs. 4 and 5, we show the condition numbers of the symmetric and the left preconditioned operators considering $\mathcal{A}$ as a periodic and a non-periodic operator respectively. We notice that the condition numbers of $P \mathcal{A} P$ and $P \mathcal{A}$ appear to be bounded when $\mathcal{A}$ is periodic. The condition number of $P \mathcal{A} P$ grows as $\mathcal{O}(N)$ whereas condition number of $P \mathcal{A}$ grows similar to the unpreconditioned operator $\mathcal{A}$ when $\mathcal{A}$ is non-periodic. We also notice from Fig. 5 that $\frac{\lambda_{3}}{\lambda_{N-2}}$ grows much slower or hardly at all for both the Fourier frame preconditioned operators $P \mathcal{A} P$ and $P \mathcal{A}$ (non-periodic operators), whereas $\frac{\lambda_{3}}{\lambda_{N-2}}$ grows as of $\frac{\lambda_{1}}{\lambda_{N}}$ for the unpreconditioned operator $\mathcal{A}\left(\lambda_{3}\right.$ is the third highest singular value and $\lambda_{N-2}$ is the third lowest singular value). Thus analyzing Figs. 3, 4, and 5 we expect that the left preconditioner and the symmetric preconditioner would perform well since most of the singular values are clustered to a constant for the both preconditioned system (though the condition number of the left preconditioner grows as of $\mathcal{A}$ when we consider a non periodic boundary condition). The condition number of $P \mathcal{A}$ and $\mathcal{A} P$ are the same, so we present experimental results of the left preconditioned system only. 

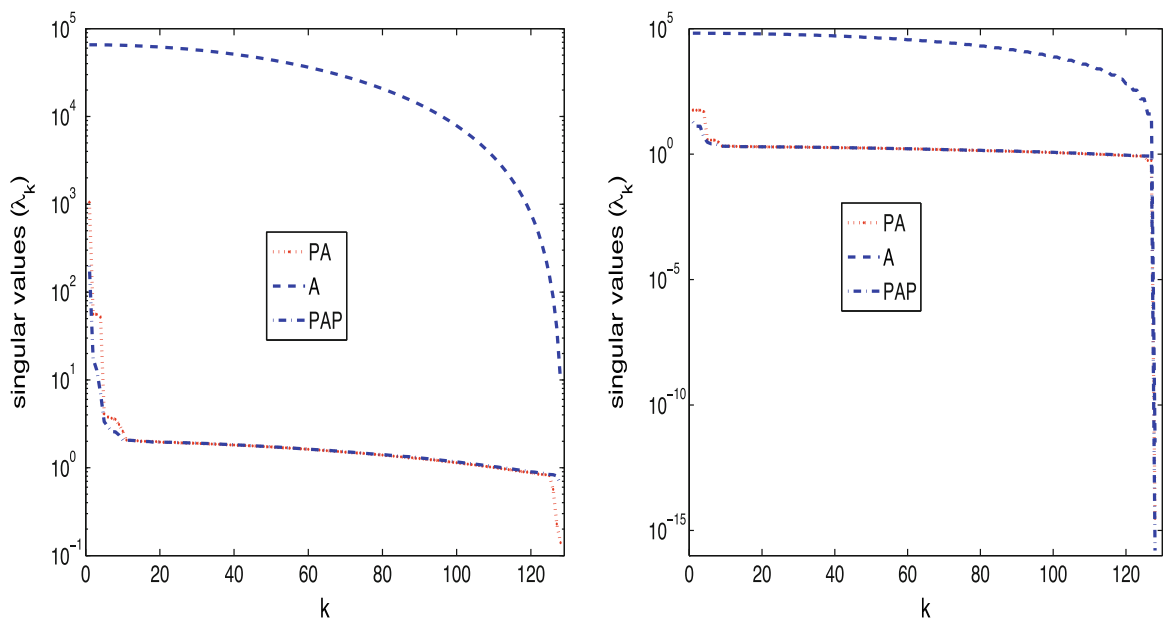

Fig. 3 Here we plot singular values of the preconditioned operators (non-periodic operator (left figure) and periodic operator (right figure)) where $K=4$ and $N_{l}=2^{6}$. From here $A$ appearing on the figures represents the discrete operator $\mathcal{A}$

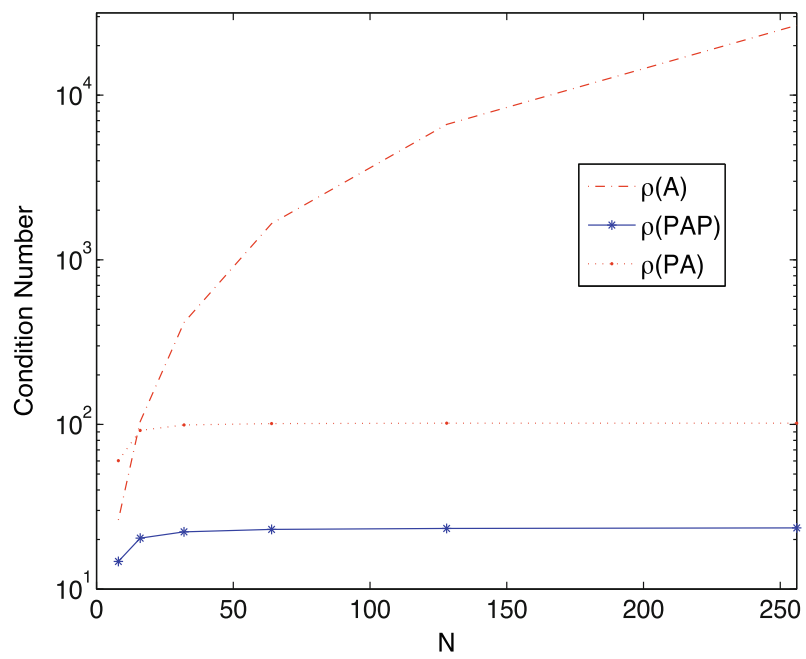

Fig. 4 Condition numbers of the operators for various choices of system sizes (periodic case)

Then we focus on solving the problem using the conjugate gradient solvers. We present the number of iterations taken to converge for three different choices of solvers considering $f(x)=\exp (2 \pi(x-0.5))$ in Fig. 6. From this experiment we notice that both the SPCG and the LBICG take a very few iterations to converge compared to the CG (for both the periodic and the non-periodic boundary value problems).

This is to note that we have considered windowed sine frames (WSF) as well for the same computations. It produces the similar results as of WFF, so one can also use WSF for the computations as well. 

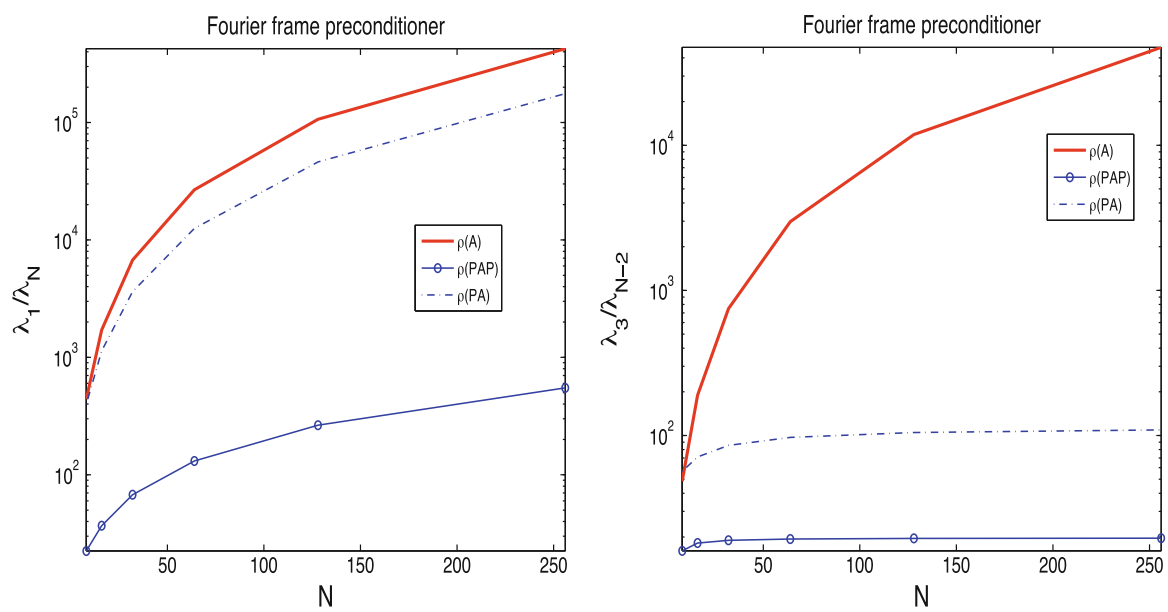

Fig. 5 Condition numbers of the windowed Fourier frame preconditioned operators (non-periodic case) for various choices of system size
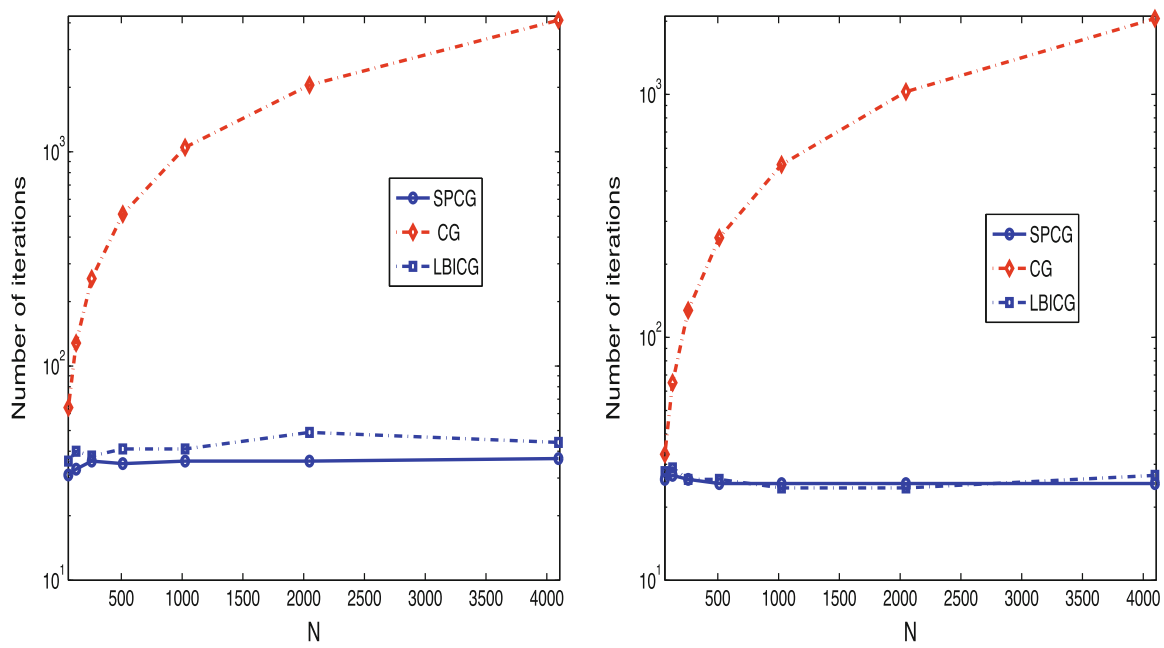

Fig. 6 Number of iterations taken by various solvers to converge (we consider 4 windows here) considering boundary conditions $(1) u(0)=0 u(1)=0$ (left figure), (2) $u(0)=u(1)$ (right figure)

We have experimented with the preconditioner considering a simple constant coefficient BVP in Example 4.1. This preconditioner is actually designed for variable coefficient problems. In the following example we aim to demonstrate the advantage of windowing for such problems.

Example 4.2 Consider the periodic BVP

$$
-\frac{d}{d x}\left(a(x) \frac{d u(x)}{d x}\right)+b(x) u(x)=f(x), \quad \forall 0 \leq x \leq 1,
$$



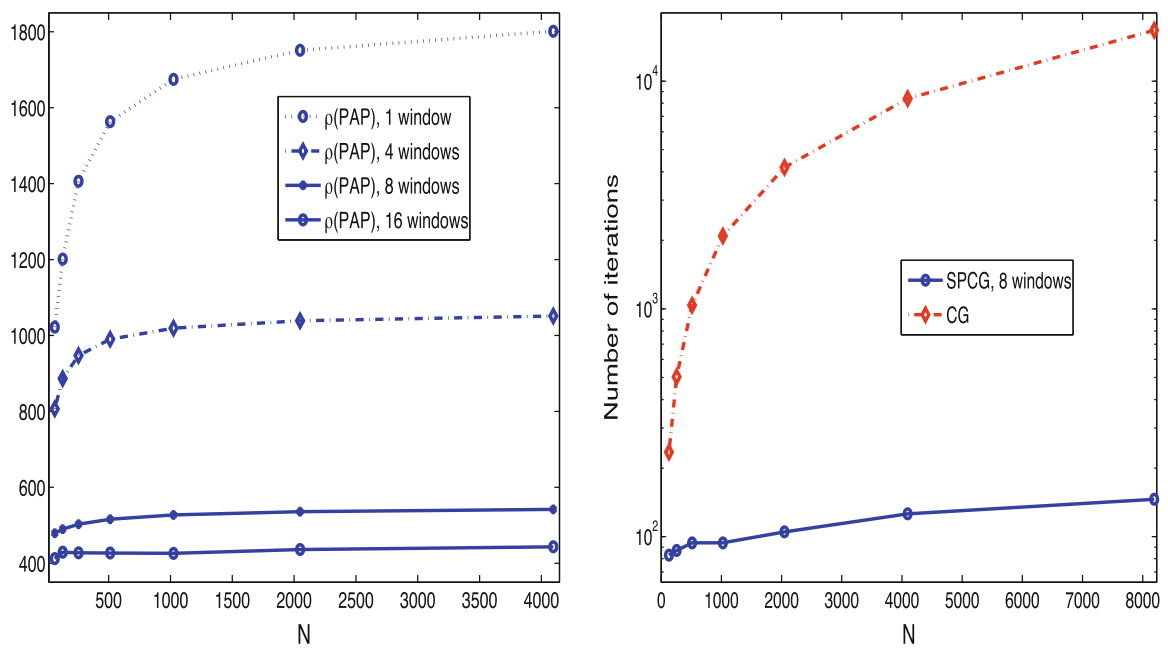

Fig. 7 We show the condition numbers for various choices of use of number of window functions. This left figure shows the efficiency of windowing by reducing the condition number. The right figure shows the efficiency of windowing by reducing the number of iterations significantly

where $a(x)=10-9.5 \cos (2 \pi x), b(x)=1$ and

$$
f(x)= \begin{cases}\exp (x) & \text { when } 0<x \leq 0.25 \\ \exp (-x) & \text { if } \quad 0.25<x \leq 1\end{cases}
$$

We use the scheme (26) and consider $t o l=10^{-10}$. In Fig. 7, we compare condition number of the discrete symmetric preconditioned variable coefficient operator in $0 \leq x \leq 1$ by varying the number of windows to cover the domain. From this experiment we notice that the more subdivisions of the domain (using windows) one considers, the smaller the condition number becomes. Then we compare the number of iterations taken by the CG method and the SPCG method (considering 8 windows to cover the periodic $[0,1]$ domain) and notice the good behavior of the preconditioned system.

Example 4.3 Consider the BVP

$$
-\frac{d}{d x}\left(a(x) \frac{d u(x)}{d x}\right)+b(x) u(x)=f(x), \quad \forall 0<x<1,
$$

with boundary conditions $u(0)=0$ and $u(1)=0$, where $b(x)=1$ for all $0<x<1, f(x)$ as a random function and

$$
a(x)= \begin{cases}\exp (x) & \text { when } 0<x<0.5 \\ \exp (-x) & \text { if } 0.5 \leq x<1 .\end{cases}
$$



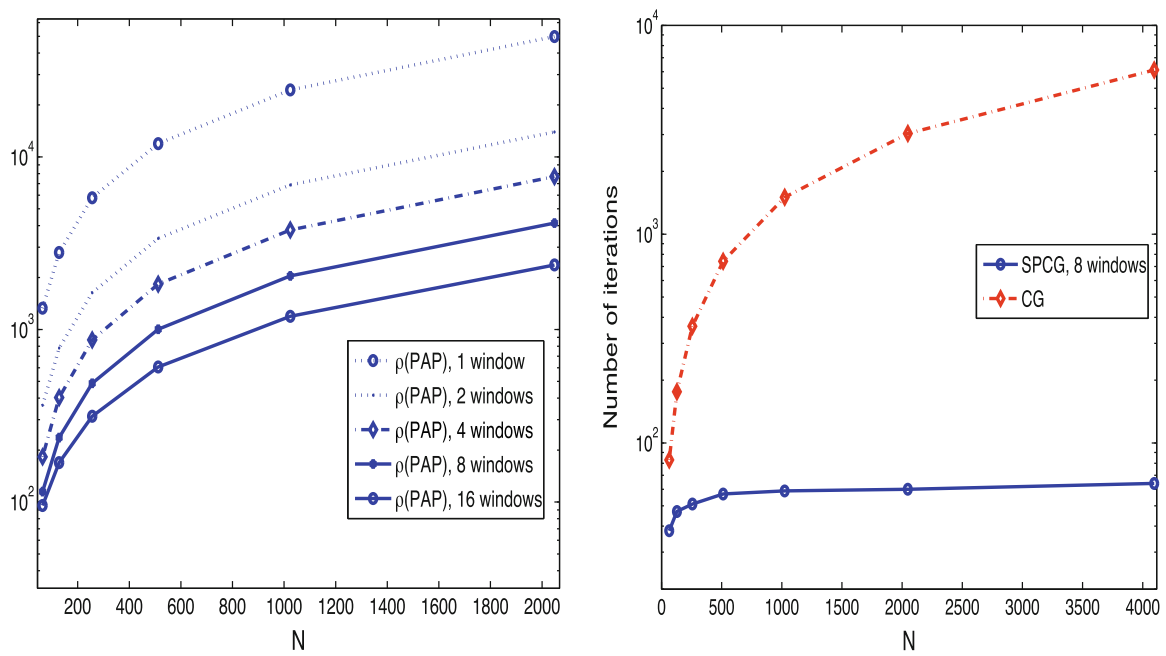

Fig. 8 The left figure shows condition numbers for different choices of windows, and the right figure shows the number of iterations taken to converge by the conjugate gradient method and the symmetric preconditioned conjugate gradient method

We use the scheme (26) and consider $t o l=10^{-10}$. In Fig. 8 we notice that the condition number keeps growing but more slowly for large $N$, while the number of iterations appears to becomes constant or close to constant.

\subsection{Two dimensional examples}

Here we apply our technique in two dimensions. We consider one example where the operator is very strong in one direction to demonstrate the advantage of using the exact symbol. For the other example we consider an operator that contains strongly varying coefficient $a(x, y)$ acting on it to demonstrate further the advantage of windowing.

Example 4.4 Consider

$$
\mathcal{L} u(x, y)=-10 \frac{\partial^{2} u(x, y)}{\partial x^{2}}-\frac{1}{10} \frac{\partial^{2} u(x, y)}{\partial y^{2}}=f(x, y)
$$

and $\Omega \subseteq \mathbb{R}^{2}$ with $\left.u\right|_{\partial \Omega}=0$. Now let $\Omega=(0,1)^{2}$ be the unit square and similar to one dimensional case, we define $\nabla y=\nabla x=h=1 / N>0$ and $\hat{x}_{i, j}=(i h, j h), i, j=$ $0,1,2, \ldots, N$. The results have been compared in Fig. 9. Here we notice that the WFF preconditioners designed with the exact symbol performs significantly better.

Example 4.5 Consider

$$
\mathcal{L} u(x, y)=-\frac{\partial}{\partial x}\left(a(x) \frac{\partial u(x, y)}{\partial x}\right)-\frac{\partial}{\partial y}\left(b(y) \frac{\partial u(x, y)}{\partial y}\right)=f(x, y),
$$



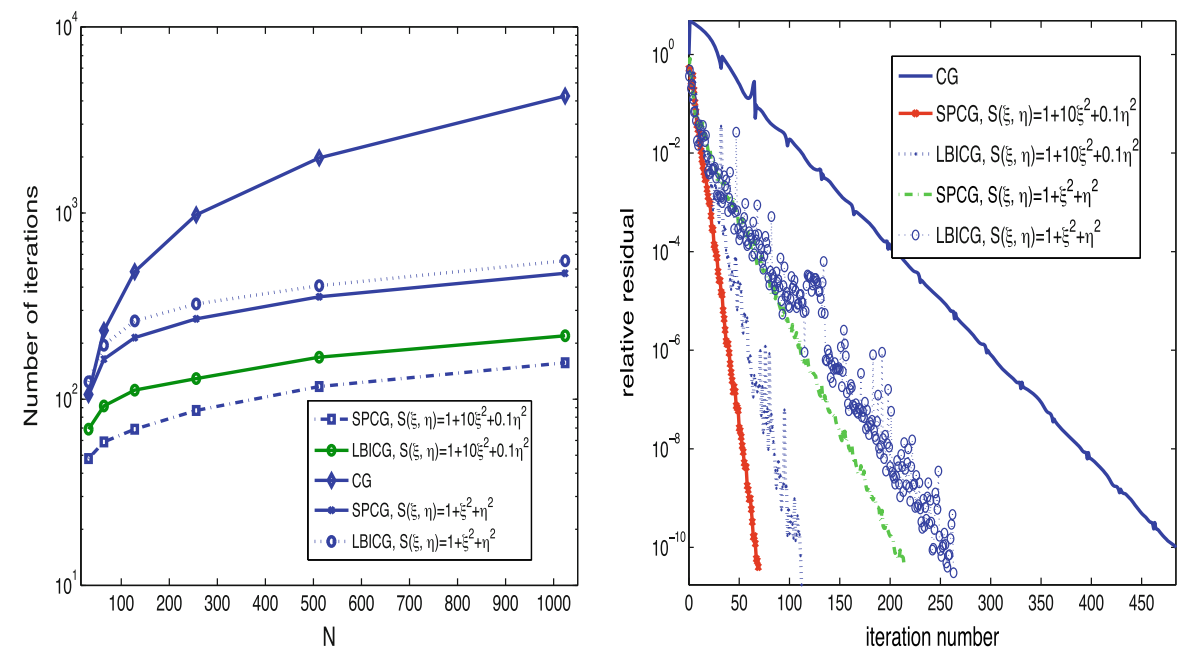

Fig. 9 In the left figure, we compare number of iterations considering the exact symbol $S(\xi, \eta)=1+$ $10 \xi^{2}+\frac{1}{10} \eta^{2}$ and an isotopic symbol $S(\xi, \eta)=1+\xi^{2}+\eta^{2}$. Here we use $N_{l, x}=N_{l, y}=2^{2: 7}$ (thus $N=2^{5: 10}$ is the number of points on each direction). In the right figure we compare relative residuals for each iteration number considering $N_{l, x}=N_{l, y}=2^{4}$. For this computation we consider $K_{x}=K_{y}=4$ windows in each directions, $f(x, y)=e^{-(x+y)}$ and $t o l=10^{-10}$

in a periodic domain $\Omega=[0,1]^{2}$, where $a(x)=10-9.5 \cos (2 \pi x)$ and $b(y)=1$. For this computations we consider symmetric preconditioned solvers only. In Fig. 10, we compare the Fourier preconditioned conjugate gradient method (one Fourier transform only, considering no windowing) and the WFF preconditioned conjugate gradient method (4 windows in each direction). Here we observe that the preconditioned solver based on the WFF performs better than the Fourier preconditioned solver.

\section{Conclusions}

We study WFFs focusing on elliptic boundary value problems and present new preconditioners based on the symbol of the operator and WFFs. From this study we conclude that the preconditioners based on WFFs work well for elliptic problems, most of the singular values clustered to a constant approximately for both preconditioned operators (SPCG and LBICG). For periodic domains, the condition number is bounded. However, when a non-periodic domain is considered, there are a few very large and a few very small singular values, which cause the condition number of the symmetrically preconditioned system to grow $\mathcal{O}(N)$ whereas the condition number of left preconditioned system grows close to $\mathcal{O}\left(N^{2}\right)$. This affects the convergence of the CG method relatively little, as it concerns only a few singular values, but a better definition of such a preconditioner on a bounded domain is clearly a question for further research.

As expected both the SPCG and the LBICG take very few iterations to converge compared to the unpreconditioned CG method. For multidimensional PDEs, if the 


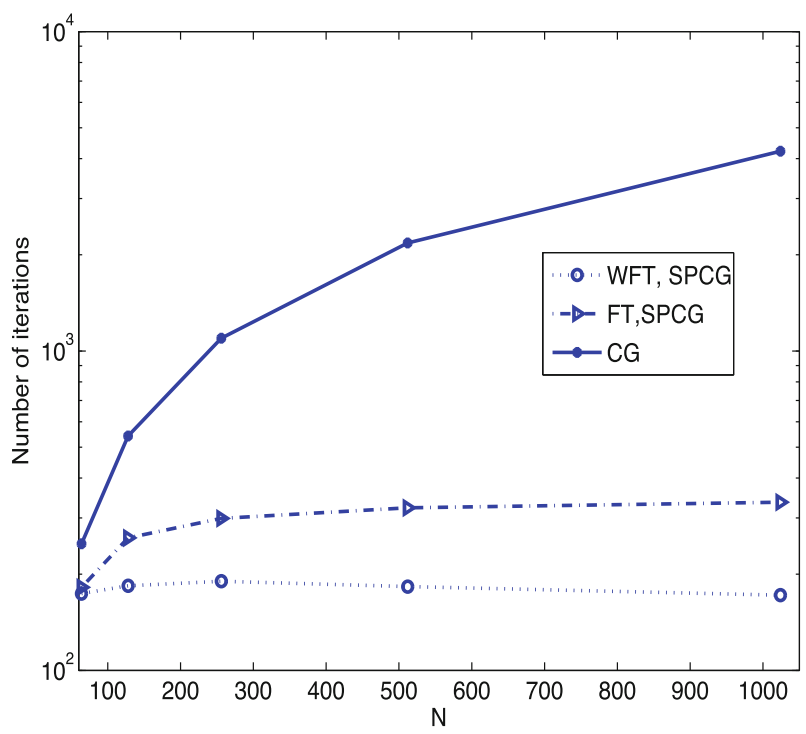

Fig. 10 We compare number of iterations considering symmetric preconditioner with 4 windows and without windows. We consider the symbol $S(\xi, \eta)=1+a(x) \xi^{2}+b(y) \eta^{2}$. Here we use $K_{x}=K_{y}=4, N_{l, x}=$ $N_{l, y}=2^{3: 7}$ (thus $N=2^{6: 10}$ is the number of points on each directions). For this computation we consider $f(x, y)=e^{-(x+y)}$ and $t o l=10^{-10}$

problem is strongly dominant in one direction then the use preconditioners based on the exact symbols is recommended. We notice that the use of reasonably many windows (while defining the preconditioner) has an advantage for PDEs with strongly varying coefficients.

\section{Appendix: Window function construction}

The window function plays an important role in the preconditioner, therefore we discuss its construction. A priori, the objective is to select a window function that satisfies the properties described in Sect. 2 and decays rapidly in the frequency domain. It is well known that smooth functions have fast decay for large frequencies [14]. So we want to form window functions that have at least some vanishing derivatives at both ends of the support. In [14] there are some general rules for constructing window functions, as well as some specific examples, we make use of this and also compare some of the example with a construction of our own. We present a schematic window function and two translations in Fig. 11.

To design $g(x)$, we consider a monotone increasing function $h(x)\left(C^{k}\right.$ or $\left.C^{\infty}\right)$ such that

$$
h(x)= \begin{cases}0 & \text { if } x \leq-1, \\ 1 & \text { if } x \geq 0\end{cases}
$$




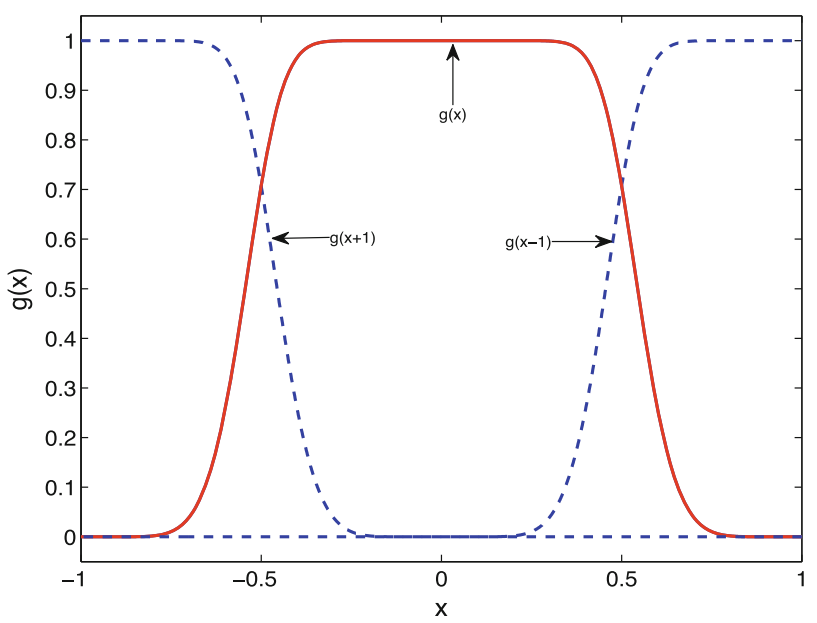

Fig. 11 The figure shows window function $g(x)$ has support $[-1,1]$, the left and the right half graphs are the translations of the function

and satisfies

$$
h^{2}(-1 / 2+y)+h^{2}(-1 / 2-y)=1, \quad-1 \leq x \leq 0 .
$$

Then to form a function $g$ with support on $\left[-v_{0}, v_{0}\right]$ we take

$$
g= \begin{cases}h\left(x / v_{0}\right) & \text { for } x<0 \\ h\left(-x / v_{0}\right) & \text { for } x \geq 0\end{cases}
$$

then (28) ensures that (4) holds. Of course a variation is possible. For example we can take a parameter $1 / 2<a<1$, and, let $g(x)=0$ for $|x|>a, g(x)=1$ for $|x|<1-a$, and $g$ described by a dilated version of $h$ for $1-a<|x|<a$.

In Fig. 12, we show some examples of monotone increasing functions. First we have a class of functions, with different $c$, given by

$$
h_{1}(x)= \begin{cases}0 & \text { if } x \leq 0, \\ \sin \left(\frac{\pi}{2} \frac{e^{-\frac{c}{x}}}{e^{-\frac{c}{x}}+e^{-\frac{c}{1-x}}}\right) & \text { if } 0<x<1 \\ 1 & \text { if } x \geq 1\end{cases}
$$

(we need to translate this backward by 1 to have a function $h$ satisfying the description above). This is constructed using the standard profile $h_{a}(x)=\frac{e^{-\frac{1}{x}}}{e^{-\frac{1}{x}}+e^{-\frac{1}{1-x}}}$, that goes smoothly from 0 to 1 on $[0,1]$ and satisfies $h_{a}(x)+h_{a}(1-x)=1$. Taking the $\sin \left(\frac{\pi}{2} h_{a}(x)\right)$ instead of $h_{a}$ transforms the property $h_{a}(x)+h_{a}(1-x)=1$ into the similar property (28) for the squares. 


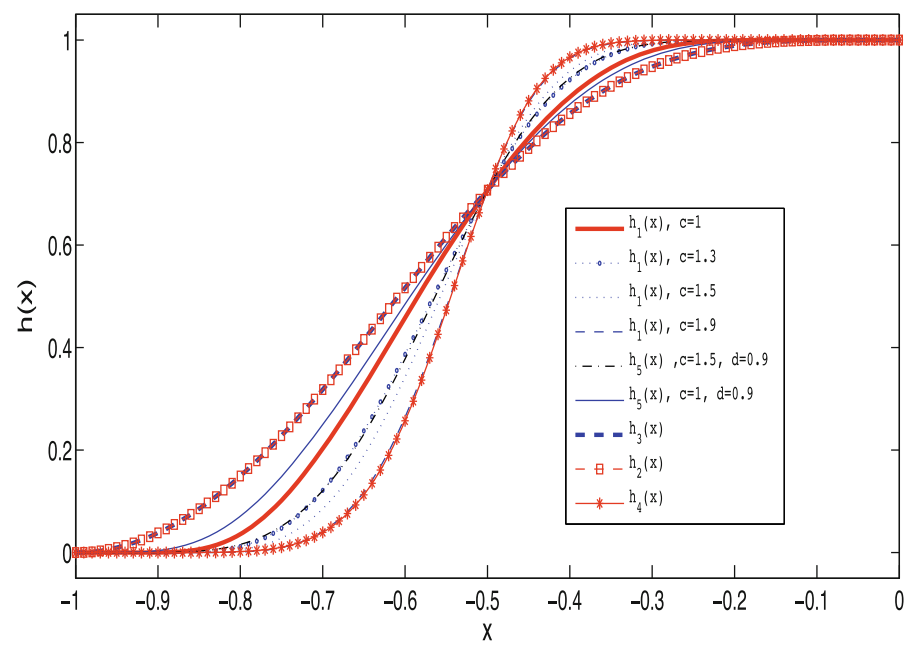

Fig. 12 The figure shows several monotone increasing functions $h(x)$ in $[-1,0]$. It is, in fact, showing left half of the window functions considered in this study

Two functions from [14] are

$$
h_{2}(x)= \begin{cases}0 & \text { if } x \leq-1 \\ \sin \left(\frac{\pi}{2} \sin ^{2}\left(\frac{\pi}{2}(1+x)\right)\right) & \text { if }-1<x<0 \\ 1 & \text { if } x \geq 0\end{cases}
$$

or

$$
h_{3}(x)= \begin{cases}0 & \text { if } x \leq-1, \\ \cos \left(\frac{\pi}{2} \sin ^{2}\left(\frac{\pi}{2} x\right)\right) & \text { if }-1<x<0, \\ 1 & \text { if } x \geq 0\end{cases}
$$

We also find another profile function following [14]. Here we consider a monotone increasing function (denote it as mallat on the legend of the figures) in $[-1,0]$

$$
h_{4}(x)= \begin{cases}0 & \text { if } x \leq-1 \\ \left.\sin \left(\frac{\pi}{4}\left(1+\sin \left(\frac{\pi}{2} \sin \left(\frac{\pi}{2} \sin \left(\frac{\pi(2 x+1)}{2}\right)\right)\right)\right)\right)\right) & \text { if }-1<x<0 \\ 1 & \text { if } x \geq 0\end{cases}
$$

It is to note that if we consider two sine functions or four sine functions inside $\sin (\pi / 4(1+f(x)))$, then the decay in the frequency domain is not as fast as of considering three sine functions defined by $h_{4}(x)$, so we decide to use $h_{4}(x)$ as a representative for this class of profile functions.

Here to find a good window function which has fast decay in frequency domain, we try several window functions $g(x)$, where $g(x)$ is defined from $g$ as given above. It can be observed that the windows with profile function $h_{1}(x)$, and the window with 


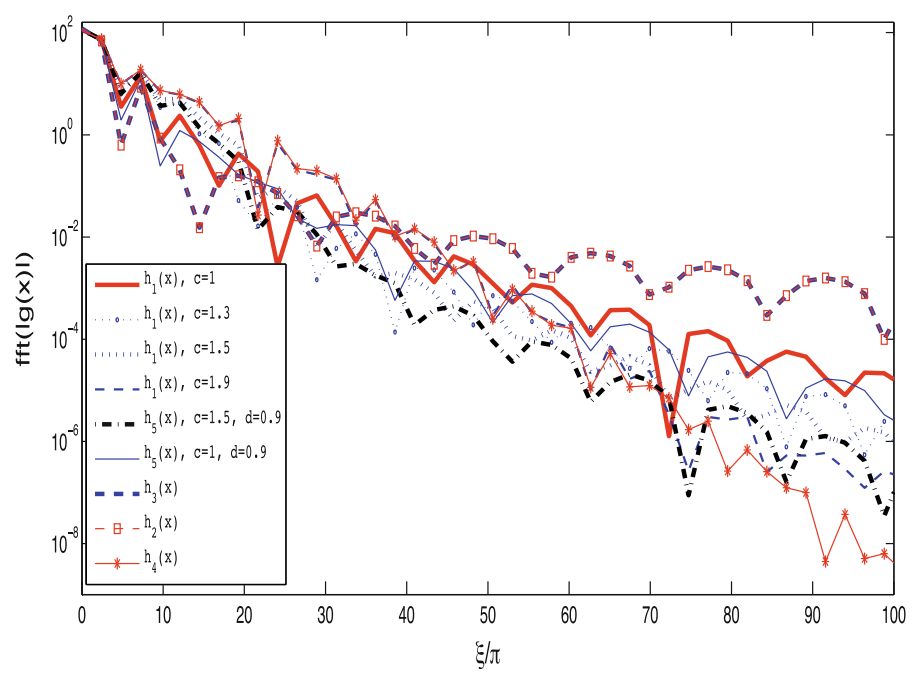

Fig. 13 The figure shows several $|\hat{g}|$ in a logarithmic scale. We consider the window function with profile function $h_{1}(x)$ with $c=[1,1.3,1.5,1.7,1.9], h_{5}(x)$ with $c=1,1.5$, and $d=0.9$, as well as considering $h_{2}(x), h_{3}(x)$ and $h_{4}(x)$

profile function $h_{4}(x)$ are smoother than the other windows and have faster decay all over the frequency domain.

We also define a dilated variant of the profile $h_{1}(x)$, effectively throwing out some of the zeros and ones on the outside of $[0,1]$. We define the dilated profile function by

$$
h_{5}(x)=h_{1}(1 / 2+d(x-1 / 2)), \quad \text { for some suitable } \quad 0<d<1 .
$$

In Fig. 13, we compare several window functions to find out which one has faster decay in the frequency domain. Analyzing the graphs, we notice that the window functions defined from monotone increasing functions $h_{2}(x)$ and $h_{3}(x)$ have slow decay in the frequency domain. All other windows have quite similar decay in the frequency domain. We observe that the window function formed by the monotone increasing function $h_{4}(x)$ and the stretched variant with $d=0.9$ and $c=1.5$ behave very well when $\xi / \pi$ is in the range of $20-60$, a region that one might still expect to be relevant, with relative size of the Fourier coefficients between $10^{-4}$ and $10^{-8}$.

Open Access This article is distributed under the terms of the Creative Commons Attribution Noncommercial License which permits any noncommercial use, distribution, and reproduction in any medium, provided the original author(s) and source are credited.

\section{References}

1. Serge, A., Patrick, G.: Pseudo-Differential Operators and the Nash-Moser Theorem. American Mathematical Society, USA (2005)

2. Nakhle, H.A.: Partial Differential Equations with Fourier Series and Boundary Value Problems, 2nd edn. Pearson, Prentice Hall, Englewood Cliffs (2005) 
3. Emmanuel, C., Laurent, D., David, D., Lexing, Y.: Fast discrete curvelet transforms. Multiscale Model. Simul. 5(3), 861-899 (electronic, 2006)

4. Raymond, H.C., Tony, F.C.: Circulant preconditioners for elliptic problems. J. Numer. Linear Algebra Appl. 1, 77-101 (1992)

5. Ke, C.: Matrix Preconditioning Techniques and Applications. Cambridge University Press, Cambridge (2005)

6. Ole, C.: An Introduction to Frames and Riesz Bases. Birkhauser, Basel (2004)

7. Albert, C.: Numerical Analysis of Wavelet Methods. Studies in Mathematics and its Applications, vol. 32. North-Holland Publishing Co., Amsterdam (2003)

8. Ingrid, D.: Ten Lectures on Wavelets. SIAM (1992)

9. Laurent, D., Lexing, Y.: Discrete symbol calculus. Technical Report arXiv:0807.0257, Jul 2008. Comments: 32 pages

10. Lawrence, C.E.: Partial Differential Equations. AMS (1998)

11. Golub, G.H., Van Loan, C.F.: Matrix Computations, 3rd edn. Johns Hopkins University Press, Baltimore (1996)

12. Herrmann, F.J., Moghaddam, P.P., Stolk, C.C.: Sparsity- and continuity-promoting seismic image recovery with curvelet frames. Appl. Comput. Harmonic Anal. 24(2), 150-173 (2008)

13. Lars, H.: The Analysis of Linear Partial Differential Operators III, Pseudo-Differential Operators. Springer, Berlin (1985)

14. Stephane, M.: A Wavelet Tour of Signal Processing, 3rd edn. Academic Press, Dublin (2009)

15. Carl, D.M.: Matrix Analysis and Applied Linear Algebra. SIAM (2000)

16. Pinchover, Y., Rubinstein, J.: An Introduction to Partial Differential Equations. Cambridge University press, Cambridge (2005)

17. Alfio, Q., Riccardo, S., Fausto, S.: Numerical Mathematics. Texts in Applied Mathematics, vol. 37. Springer, Berlin (2000)

18. Ruzhansky, M., Turunen, V.: On the fourier analysis of operators on the torus. Oper. Theory Adv Appl Birkhuser Basel 172, 87-105 (2006)

19. Trottenberg, U., Oosterlee, C.W., Schüller, A.: Multigrid. Academic Press San Diego (2001). With contributions by A. Brandt, P. Oswald and K. Stüben

20. Turunen, V., Vainikko, G.: On symbol analysis of periodic pseudodifferential operators. Z. Anal. Anw. 172, 9-22 (1998)

21. Wong, M.W.: An Introduction to Pseudodifferential Operators. World Scientific, Singapore (1991) 